\title{
The evolution of antibody response in armadillos inoculated with Mycobacterium leprae
}

\author{
A R VADIEE, E HARRIS \& E J SHANNON \\ Laboratory Research Branch, Gillis W. Long Hansen's Disease \\ Center, Carville, Louisiana 70721, USA
}

Accepted for publication 15 January 1990

\begin{abstract}
Summary Plasma from 30 armadillos (Dasypus novemcinctus) was collected prior to inoculation and at approximately 3-month intervals for a period of 1-3 years. These animals were inoculated intravenously with $6.1 \times 10^{8} \pm 2 \times 10^{8}(\mathrm{x} \pm \mathrm{SD})$ armadillo-derived Mycobacterium leprae. These samples were analysed for antibodies of IgM and IgG class to phenolic glycolipid-I (PGL-I) and to sonicated $M$. leprae components using ELISA and immunoblotting techniques, respectively. We had previously observed among a group of 11 armadillos, that some animals produced and maintained a high IgG antibody response to PGL-I. In this study, an animal's ability to produce and maintain an elevated IgG anti-PGL-I response was significantly correlated with their ability to delay dissemination of the infection and their ability to survive longer. When the animals were moribund, a significant decrease in the IgG anti-PGL-I absorbance value was observed. The detection of PGL-I in the plasma samples collected from moribund armadillos suggested that high concentrations of PGL-I in the plasma may have contributed to a drop in absorbance values by the formation of non-lattice-type immune complexes in vivo.

As detected by immunoblotting, the $\mathrm{IgM}$ and $\mathrm{IgG}$ response to antigens derived from sonically disrupted $M$. leprae was directed towards molecules with broad bands of immunoreactivity ranging from $21-$ to $45-\mathrm{kDa}$. There were no distinguishing features of these antibody responses among armadillos as was evident with the IgG anti-PGL-I responses.
\end{abstract}

\section{Introduction}

Leprosy in man has a broad clinical manifestation determined by the host parasite relationship. The spectrum of variation in resistance to infection with Mycobacterium leprae ranges from highly resistant (tuberculoid leprosy) to highly susceptible (lepromatous leprosy). ${ }^{1}$ Nine-banded armadillos (Dasypus novemcinctus) are highly susceptible to infection with $M$. leprae ${ }^{2}$ and when inoculated, they develop primarily lepromatous-type disease. ${ }^{3}$ Therefore, armadillos are considered by many investigators to be a good 
experimental model for studying lepromatous leprosy. One characteristic of human lepromatous leprosy, as well as that of infected armadillos, is the production of large amounts of antibodies against mycobacterial antigens. ${ }^{4,5}$ These antibodies have played a significant role in describing the immunogenic structure of $M$. leprae. Components such as antigen $7,{ }^{6}$ cell-wall polysaccharides, ${ }^{4}$ proteins ${ }^{4,7}$ and phenolic glycolipid-I (PGL-I) ${ }^{8-10}$ are recognized as antibody evoking immunogens in both humans and armadillos.

Due to difficulties in detection of subclinical leprosy in man, a description of the evolution of antibodies to components of $M$. leprae among patients in the presence or absence of chemotherapy has not been reported. Armadillos experimentally inoculated with $M$. leprae offer an opportunity to describe the evolution of antibodies to $M$. leprae following exposure to known quantities of $M$. leprae and in the absence of chemotherapy.

This study describes the evolution of armadillo IgM and IgG antibodies to PGL-I and sonicated $M$. leprae using ELISA and immunoblotting.

\section{Methods}

\section{ARMADILLO PLASMA}

Plasma from 30 nine-banded armadillos was collected prior to inoculation (day 0 ) and at approximately 3 -month intervals for a period of $1-3$ years. The conditions for adopting the animals into the colony and their maintenance have been described previously. ${ }^{5}$ These animals were inoculated intravenously with $6 \cdot 1 \times 10^{8} \pm 2 \times 10^{8}(\mathrm{x} \pm \mathrm{SD})$ armadillo-derived M. leprae.

\section{ENZYME-LINKED IMMUNOSORBENT ASSAY (ELISA)}

One hundred and ninety-two plasma samples were analysed by means of ELISA. The ELISA, for detection of armadillo IgM and IgG antibodies to PGL-I, was carried out as described previously. ${ }^{10}$ Armadillo-derived PGL-I was provided by Dr Brennan (NIH Contract AI-52582, Colorado State University).

\section{PREPARATION OF $M$. LEPRAE EXTRACTS}

$M$. leprae were provided by Dr P Brennan as irradiated, lyophilized bacilli purified from the lymph nodes of infected armadillos. $M$. leprae ( $10 \mathrm{mg}$ dry weight) was suspended in $3 \mathrm{ml}$ of $0.01 \mathrm{M}$ phosphate buffered saline (PBS) $\mathrm{pH} \mathrm{7.0.} \mathrm{The} \mathrm{organisms} \mathrm{were} \mathrm{disrupted} \mathrm{by}$ sonication on ice for $30 \mathrm{~min}$ at $150 \mathrm{~W}$ on a sonifier cell disrupter with a temperature control probe (Model W1851, Heat System, Ultrasonics, Inc., North Tonawanda, New York, USA) adjusted for $8^{\circ} \mathrm{C}$. The sonicated material was aliquoted and stored at $-20^{\circ} \mathrm{C}$ until used.

\section{IMMUNOBLOT}

M. leprae components $(3.33 \mathrm{mg} / \mathrm{ml})$ were separated by SDS-PAGE and electrophoretically transferred at constant voltage $(10 \mathrm{~V})$ for $18 \mathrm{hr}$ to BA 85 nitrocellulose paper (NCP) (Schleicher and Schuell, Inc., Keene, New Hampshire, USA) in a tris-glycine-methanol buffer, $\mathrm{pH} 8 \cdot 0 .{ }^{11}$ After transfer, the NCP was incubated at room temperature (RT) for 45 
min in 0.01 M PBS containing $3 \%$ bovine serum albumin (BSA). The NCP was then incubated with armadillo plasma at 1:50 dilution for $1 \mathrm{hr}$ at RT. The NCP was washed five times for $5 \mathrm{~min}$ each in $0.01 \mathrm{M}$ PBS containing $0.05 \%$ Tween 20 . It was then reacted with rabbit antiarmadillo gamma-chain at $1: 1000$ dilution or with rabbit antihuman uchain at 1:50 dilution. The NCP strips were washed and treated with peroxidase conjugated goat antirabbit immunoglobulins ( $\operatorname{Ig} M+\operatorname{IgG}+\operatorname{IgA})$ (Cappel Laboratories, Downington, Pennsylvania, USA) at 1:1000 dilution. Next, the NCP was washed and developed with $\mathrm{H}_{2} \mathrm{O}_{2}$ /horseradish peroxidase (HRP) colour development reagent for 10 min at RT as described by the manufacturer (Bio-Rad, Richmond, California, USA). The reaction was stopped by transfer of the NCP to $5 \%$ acetic acid in deionized $\mathrm{H}_{2} \mathrm{O}$.

\section{EXTRACTION OF PGL-I FROM ARMADILLO PLASMA}

Plasma samples $(0 \cdot 3-0 \cdot 5 \mathrm{ml})$ were added dropwise to $5 \mathrm{ml}$ of $95 \%$ ethanol, resulting in the formation of a whitish precipitate. The samples were centrifuged at $1380 \times G$ for $10 \mathrm{~min}$ and the supernatants were decanted. The excess ethanol was removed by inverting the tubes and allowed to drain for $10 \mathrm{~min}$. Lipids were extracted from the residue with $5 \mathrm{ml}$ chloroform:methanol $(2: 1 \mathrm{~V} / \mathrm{V})$ at $50^{\circ} \mathrm{C}$ overnight. The extracts were centrifuged at $1380 \times G$ for $10 \mathrm{~min}$. The supernatants were removed and residues were washed with $5 \mathrm{ml}$ of chloroform:methanol $(2: 1)$ and re-centrifuged. The two supernatants from each sample were combined and taken to dryness at $50^{\circ} \mathrm{C}$ under nitrogen. The procedure for extracting the PGL-I from the total lipid extract was based on that of Hunter et al. ${ }^{12}$ Briefly, dried lipids were dissolved in chloroform and applied to a silicic acid:celite column $(2: 1 \mathrm{~V} / \mathrm{V})$. The column was successively eluted with two bed volumes each chloroform, $2 \%$ methanol in chloroform and 5\% methanol in chloroform. The $2 \%$ and $5 \%$ eluates were combined and taken to dryness under nitrogen at $50^{\circ} \mathrm{C}$ and analysed for PGL-I by thin-layer chromatography (TLC) and ELISA.

For TLC, samples were applied to high performance silicel gel plate (Sigma, St Louis, $\mathrm{MO})$ and run in a solvent system composed of ether: acetone $(8: 2, \mathrm{~V} / \mathrm{V})$. The plates were air dried and PGL-I was located by spraying the plates with orcinol:sulphuric acid reagent and heating in a drying oven at $110^{\circ}-115^{\circ} \mathrm{C}$ for $3-5 \mathrm{~min}$. PGL-I at 5-10 $\mu \mathrm{g}$ served as the standard marker.

For ELISA, the samples were suspended in $100 \mu \mathrm{l}$ of $0.05 \mathrm{M}$ carbonate-bicarbonate buffer $\mathrm{pH} 9 \cdot 2$. The samples were further diluted $1: 4$ in the carbonate-bicarbonate buffer and were used for coating the microtiter plate $\left(50 \mu \mathrm{l} /\right.$ well). The plate was incubated at $4{ }^{\circ} \mathrm{C}$

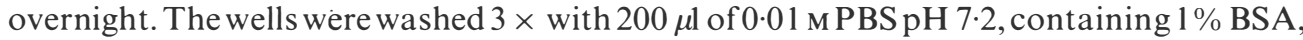
and blocked by incubation with $100 \mu \mathrm{l}$ of $0.01 \mathrm{M}$ PBS containing 5\% BSA at RT for $1 \mathrm{hr}$. The contents were aspirated and $50 \mu \mathrm{l}$ of monoclonal antibody F8b4 (kindly provided by Thomas Buchanan) diluted 1:500 in 1\% BSA/PBS was added to all wells. The plate was incubated at RT for $1 \mathrm{hr}$. After washing, $50 \mu \mathrm{l}$ of peroxidase-conjugated rabbit antimouse IgM ( $\mu$-chain-specific (Cappel Laboratories, Downington, Pennsylvania, USA) at $1: 500$ dilution was added per well and incubated for $1 \mathrm{hr}$ at RT. After washing the plate, $50 \mu 1$ of $0.04 \mathrm{mg} / \mathrm{ml}$ solution of orthophenylene diamine (Sigma Chemical Co., St Louis, MO, USA) containing $0.02 \% \mathrm{H}_{2} \mathrm{O}_{2}$ in $0.02 \mathrm{M}$ sodium acetate buffer, $\mathrm{pH} 5 \cdot 5$, was added to each well. The plate was incubated at RT for $10 \mathrm{~min}$, and the reaction was stopped by adding $5 \mathrm{M} \mathrm{HCl}(50 \mu \mathrm{l} /$ well). Absorbance at $492 \mathrm{~nm}$ was read with a spectrophotometer (Titertek Multiscan, Flow Laboratories, Richmond, Virginia, USA). Wells not coated 
with antigen served as negative control. For positive controls, $50 \mu \mathrm{l}$ of known concentrations of PGL-I ranging from 0.025 to $80 \mu \mathrm{g} / \mathrm{ml}$ were used for coating the wells.

\section{STATISTICAL ANALYSIS}

Results were analysed for statistical significance on a Hewlett-Packard 9845T computer using the one tailed paired $t$-test and $t$-test. Values of $P<0.05$ were considered to be statistically significant.

\section{Results}

\section{ARMADILLO ANTIBODIES TO PGL-I}

The evolution of IgM and IgG anti-PGL-I response among a group of 30 armadillos is shown in Figure 1. Compared to the baseline ( 0 day), the anti-PGL-I response increased significantly up to approximately 450 days postinoculation (PI) for IgM and up to approximately 630 days PI for IgG. When line drawing graphs of the thirty individual armadillos IgM and IgG anti-PGL-I responsiveness $v s$ days postinoculation with $M$. leprae was plotted, a homogenous clustered IgM response emerged; whereas a scattered IgG anti-PGL-I response was observed (data not shown). Although the IgG anti-PGL-I responsiveness was heterogenous, some animals clearly had higher absorbance values for IgG antibodies to PGL-I as compared to others. Animals with high absorbance values for IgG anti-PGL-I antibodies, despite the progression of their disease, maintained their high responsiveness. To summarize these data, animals were grouped into high and low IgG anti-PGL-I responders. The criteria for grouping these animals was based on absorbance

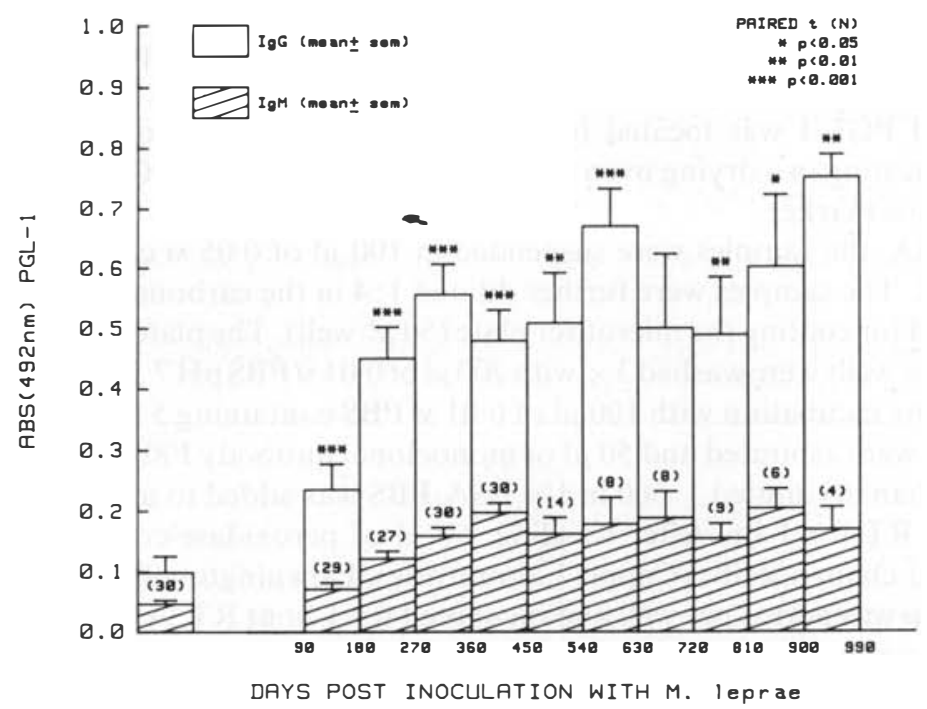

Figure 1. IgM and IgG anti-PGL-I response of 30 armadillos during course of experimental infection. Asterisks indicate IgG anti-PGL-I absorbance values significantly different from preceding absorbance values (paired $t$ test). 
values observed at 360 days postinoculation. Eleven high IgG anti-PGL-I responders (Figure 2, Group A) had absorbance values within a mean \pm 2 , SEM of $0 \cdot 745 \pm 0 \cdot 136$; whereas, nineteen low IgG anti-PGL-I responders had absorbance values within a mean \pm 2 , SEM of $0 \cdot 212 \pm 0 \cdot 056$ (Figure 2, Group B).

When animals in Group A were compared to those in Group B in relation to their longevity after inoculation with $M$. leprae, it was shown that animals in Group A survived for a longer period (1051 days vs 563 days) (Table 1). The armadillos used for this study were also regularly examined histologically for the presence of acid-fast bacilli (AFB) in

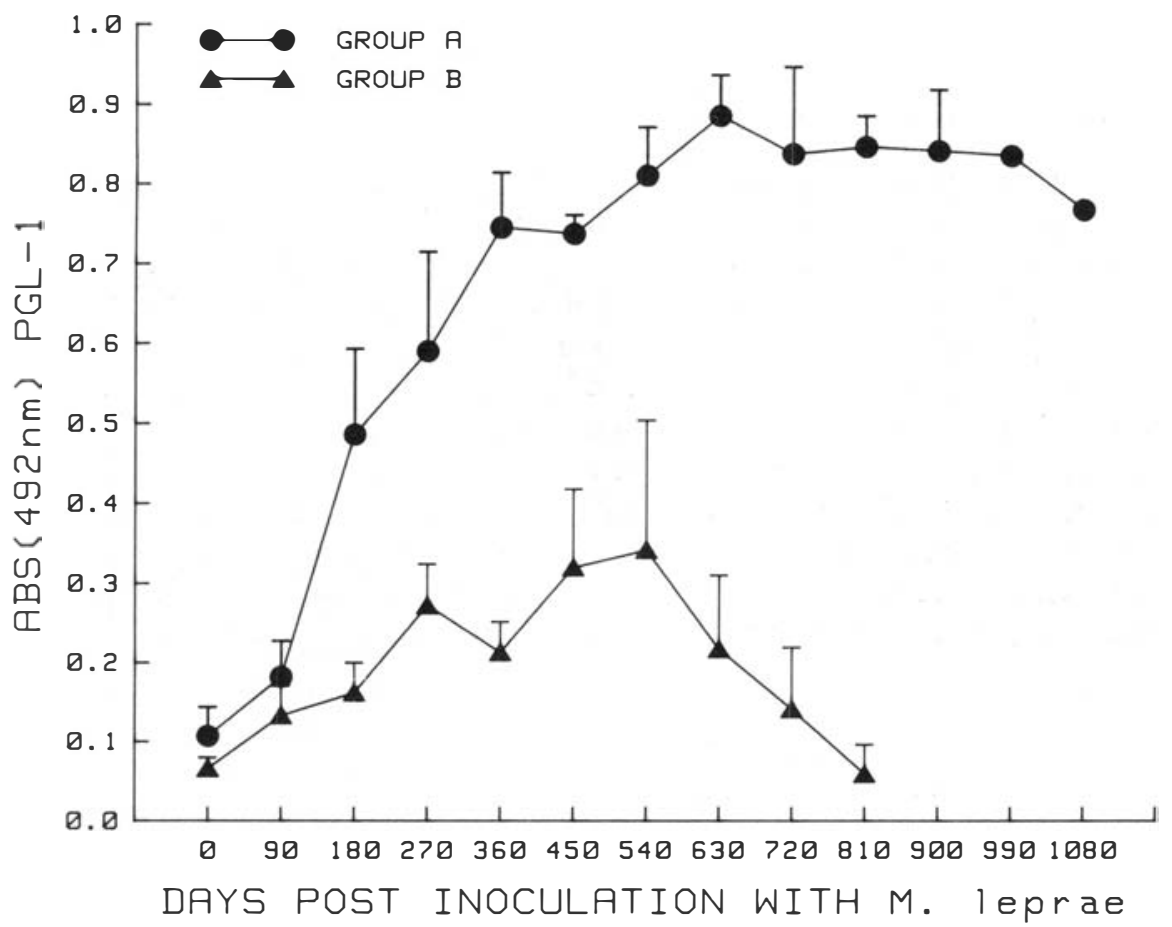

Figure 2. Kinetics of IgG anti-PGL-I mean \pm SEM. Animals in Group A $(n=11)$; animals in Group B $(n=19)$.

Table 1. Comparison of Ig G absorbance values to PGL-I with longevity and time until the appearance of AFB in ear biopsies among 30 armadillos inoculated with $M$. le prae

\begin{tabular}{lccc}
\hline & $\begin{array}{c}\text { IgG ELISA at } \\
360 \text { days post } \\
\text { inoculation } \\
\text { (OD at } 492 \mathrm{~nm})\end{array}$ & $\begin{array}{c}\text { Longevity } \\
\text { (days) }\end{array}$ & $\begin{array}{c}\text { Time to AFB } \\
\text { appearance in } \\
\text { ear biopsy } \\
\text { (days) }\end{array}$ \\
\hline $\begin{array}{l}\text { High IgG anti-PGL-I responder } \\
(\mathrm{N}=11)\end{array}$ & $0.745 \pm 0.068^{*}$ & $1051 \pm 65$ & $717 \pm 139$ \\
$\begin{array}{l}\text { Low IgG anti-PGL-I responder } \\
(\mathrm{N}=19)\end{array}$ & $0.212 \pm 0.038$ & $563 \pm 41$ & $417 \pm 39$ \\
& & $P<0 \cdot 0^{\circ}$ & $P<0.01 \dagger$ \\
\hline
\end{tabular}

* Values expressed as mean $\pm \mathrm{SEM}$.

$\dagger t$-test, one tailed. 
ear biopsies as a means of detecting dissemination of the disease. By this criterion, Group A animals delayed the dissemination of the disease as compared to those in Group B (717 days $v s 417$ days). These differences as represented in Table 1 were statistically significant $(P<0.01)$.

The animals from both groups were sacrificed when they exhibited a heavy dissemination of $M$. leprae infection as determined by histological examination of their ear biopsies. Interestingly, the bacterial load in organs like the spleen, liver and lymph nodes in both populations was quite similar. However, one animal from Group A is still alive and information regarding its bacterial load is not available.

\section{ARMADILLO ANTIBODIES TO M. LEPRAE COMPONENTS}

Plasma samples collected during the first year postinoculation with $M$. le prae from a total of 9 animals, 4 from Group A and 5 from Group B (Figure 2), were selected and used in immunoblot analysis. The results obtained from both groups indicated a predominant $\mathrm{IgM}$ and $\mathrm{IgG}$ antibody response to be directed towards $M$. leprae components with molecular weights ranging from 21 - to $45-\mathrm{kDa}$. These components were also reactive with antibody of the IgM and IgG class in normal plasma ( 0 day) in $8 / 9$ and $2 / 9$ armadillos, respectively. Representative data as derived by selection of a given animal from each group is presented in Figure 3. One unique armadillo from Group B produced antibodies of $\mathrm{IgM}$ and $\mathrm{IgG}$ class to multiple components of $M$. leprae with time postinoculation (Figure 4). Multiple bands of immunoreactivity ranging from approximately 5-kDa to $92-$ $\mathrm{kDa}$ were observed when blots were developed for IgG response using a plasma sample collected at 363 days PI. IgM response appeared as broad diffuse bands of immunoreactivity. IgM response to the $65-\mathrm{kD}$ a protein was observed among most animals throughout the course of infection. IgG response to the $65-\mathrm{kDa}$ protein was mostly observed during the later phase of infection. The animal with antibody activity to multiple components of M. leprae showed a very strong IgG response to the $65-\mathrm{kDa}$ protein (Figure 4).

ANALYSIS OF COMPONENTS EXTRACTED FROM ARMADILLO PLASMA BY TLC AND ELISA

Eight armadillos, 3 from Group A and 5 from Group B were selected for this phase of the study. Plasma samples of each animal were selected at 4 periods and analysed for presence of PGL-I. These included the plasma collected prior to inoculation with $M$. leprae (normal) and 3 which were collected during the early, mid and late phase of infection. Seven out of the 8 plasma samples which were collected during the latter phase of infection contained components which had migration patterns similar to that of standard PGL-I using TLC. These included 2 animals from Group A (animals 1 and 2) and 5 animals from Group B (animals 4-8) (Table 2). Furthermore, plasma samples of 2 animals in Group B (animals 5 and 6) which were collected during the mid-phase of infection also had components with migration patterns similar to that of PGL-I (Table 2). A representative migration pattern of samples collected during the latter phase of infection from animals in Group A and B using TLC is shown in Figure 5. A broad staining band was observed at the region characteristic to that of standard PGL-I. It is clear that the RF value of tested samples are slightly smaller than that of standard PGL-I. However, it is believed that this 


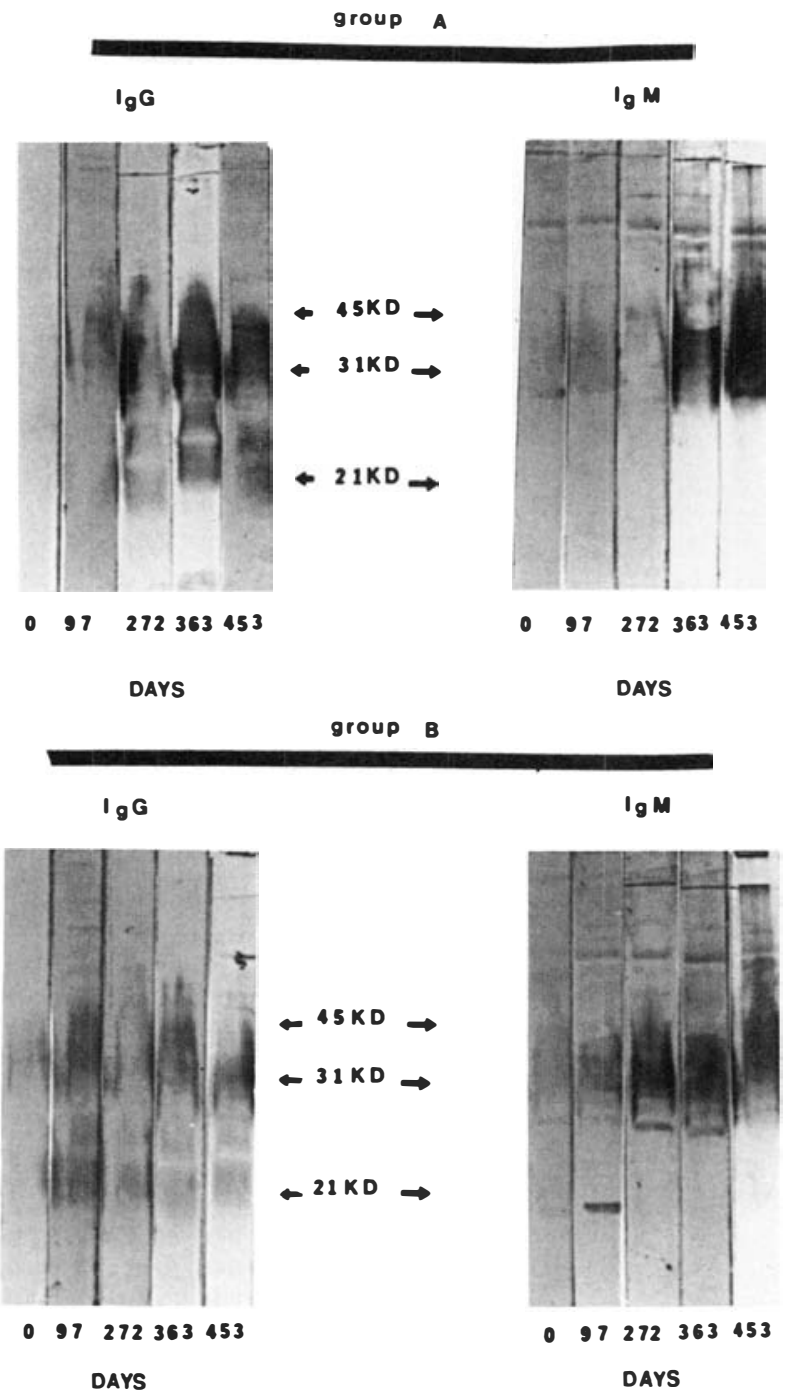

Figure 3. Demonstration of antigenic components of $M$. leprae eliciting IgM and IgG antibody response. A representative immunoblot characteristic common to animals in Group A and Group B are shown here. Sonicated $M$. leprae components were separated by SDS-PAGE and electrophoretically blotted onto NCP. NCP was incubated with armadillo serum (1:50 dilution), washed, and treated with class-specific antisera (rabbit antiarmadillo gamma-chain at $1: 1000$ dilution, rabbit antihuman $\mu$-chain at $1: 50$ dilution). The NCP strips were washed and treated with peroxidase-conjugated goat antirabbit immunoglobulin $(\operatorname{IgM}+\operatorname{IgG}+\operatorname{IgA})$ at $1: 1000$ dilution.

discrepancy may be due to the association of other components with PGL-I. The TLC results for each plasma sample were visually scored and were presented in Table 2.

To confirm that the components extracted from the plasma were PGL-I, we analysed their immunoreactivity in ELISA by using a specific monoclonal antibody F8b4 to PGLI. The samples having migration patterns similar to that of PGL-I were shown to be 


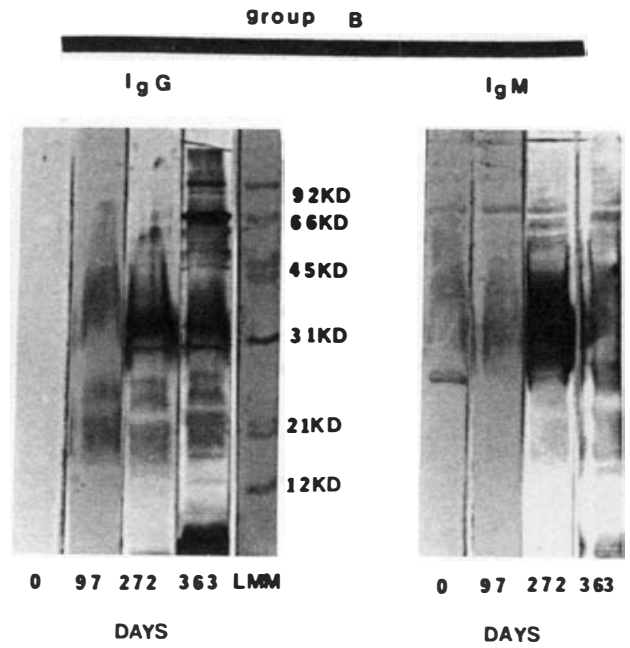

Figure 4. Demonstration of antigenic components of $M$. leprae eliciting IgM and IgG antibody response. Technical detail is similar to that of Figure 3.

Table 2. Results obtained from thin layer chromatography (TLC) and ELISA analysis of the components extracted from armadillo plasma

\begin{tabular}{|c|c|c|c|c|c|c|c|c|}
\hline \multicolumn{5}{|c|}{ TLC* } & \multicolumn{4}{|c|}{ ELISA $†(A B S 492$ nm) } \\
\hline \multirow{2}{*}{$\begin{array}{l}\text { Animal } \\
\text { number }\end{array}$} & \multirow{2}{*}{$\begin{array}{c}\text { Normal } \\
\text { (pre-infection) }\end{array}$} & \multicolumn{3}{|c|}{ Infection } & \multirow{2}{*}{$\begin{array}{c}\text { Normal } \\
\text { (pre-infection) }\end{array}$} & \multicolumn{3}{|c|}{ Infection } \\
\hline & & Early & Mid & Late & & Early & Mid & Late \\
\hline 1 & - & - & - & + & $0 \cdot 0$ & 0.04 & 0.09 & $0 \cdot 10$ \\
\hline 2 & - & - & - & + & $0 \cdot 0$ & 0.05 & $0 \cdot 20$ & $0 \cdot 14$ \\
\hline 3 & - & - & - & - & $0 \cdot 0$ & 0.00 & 0.00 & 0.00 \\
\hline 4 & - & ND $\ddagger$ & - & + & 0.0 & 0.00 & 0.07 & $0 \cdot 19$ \\
\hline 5 & - & - & + & + & $0 \cdot 0$ & $0 \cdot 12$ & $0 \cdot 17$ & $0 \cdot 14$ \\
\hline 6 & - & - & + & + & 0.0 & 0.02 & $0 \cdot 06$ & $0 \cdot 14$ \\
\hline 7 & - & ND & - & + & 0.0 & ND & $0 \cdot 01$ & 0.08 \\
\hline 8 & - & - & - & + & $0 \cdot 0$ & 0.00 & 0.00 & $0 \cdot 10$ \\
\hline
\end{tabular}

* TLC values were scored subjectively based on intensity of the stained band.

†ELISA absorbance values for normal plasma were considered as base line, and is presented here as zero. Subsequent values were derived by their subtraction from the preceding normal plasma value.

$\ddagger \mathrm{ND}=$ not done.

reactive with MAB F8b4 and, therefore, identified as PGL-I (Table 2). A sample collected during the early phase of infection from animal 5 was positive for PGL-I in ELISA, but not TLC.

\section{Discussion}

In the present study, the evolution of IgG and IgM antibodies of armadillos to PGL-I and to sonicated $M$. leprae were analysed using ELISA and immunoblotting. 
TLC

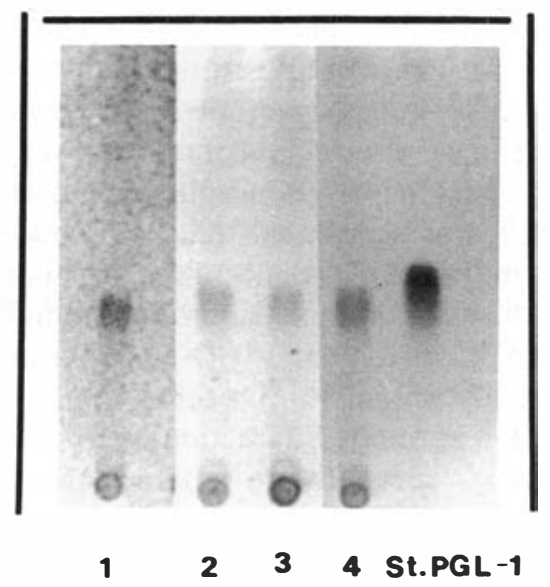

Figure 5. Comparison of the migration pattern of the component extracted from armadillo plasma with that of a standard PGL-I, using TLC. Samples were applied to high performance silicel gel plate and run in a solvent system composed of ether:acetone $(8: 2, \mathrm{~V} / \mathrm{V})$. Samples were located using orcinol:sulphuric acid reagent. Lanes 1-4, components extracted from plasma of 4 armadillos during the latter phase of infection.

Longitudinal IgM and IgG responses of a larger group of armadillos to PGL-I were performed in order to confirm our previous findings ${ }^{10}$ and to distinguish the high and low IgG anti-PGL-I responders for their further characterization regarding their antibody response to other $M$. leprae components.

The results of the present study substantiate our previous findings, ${ }^{10}$ in that $\operatorname{IgM}$ antiPGL-I absorbance values increase with time postinoculation. This response was quite homogeneous and it persists throughout the course of infection, probably due to the availability of a continuous source of antigen. In comparison to the $\operatorname{IgM}$ response, the absorbance values for IgG anti-PGL-I were considerably higher and the response was heterogeneous. As reported previously, ${ }^{10}$ and also in this study, due to the heterogeneity in absorbance values for IgG anti-PGL-I, we were able to separate armadillos into two groups. Group A represents those with high absorbance values for IgG anti-PGL-I response, whereas Group B represents the armadillos with low absorbance values for IgG anti-PGL-I. The animals in Group A, in relation to those in Group B, were capable of delaying the dissemination of $M$. leprae infection as measured by time to appearance of AFB in ear biopsies. These animals also had a longer life span. These differences existing among the two groups were statistically significant when a larger group of animals was analysed.

In human studies, Levis et al. ${ }^{13}$ have reported high IgG anti-PGL-I in some patients with tuberculoid (BT) leprosy, a form of leprosy that is associated with controlling the infection. When Gormus, et al. ${ }^{14}$ analysed longitudinal serum samples of $M$. lepraeinfected sooty mangabey monkeys for antibodies of $\mathrm{IgG}$ and $\mathrm{IgM}$ class to PGL-I, they reported that high IgG and low IgM anti-PGL-I levels are associated with less severe disease. Therefore, there may be an association between elevated IgG anti-PGL-I responses and upgrading of immunological responsiveness to $M$. leprae. 
We had previously observed a sharp drop in absorbance values for IgG anti-PGL-I among 4 of 11 animals during the latter phase of infection and at a time when the animals were moribund. ${ }^{10}$ Significant decrease in IgG anti-PGL-I levels have also been described in $M$. leprae-inoculated sooty mangabey monkeys. ${ }^{14}$ These usually preceded and/or corresponded to periods of clinical progression of the leprosy symptoms. Since the drop in absorbance values was in parallel to the systemic dissemination of infection as manifested by bacteria in buffy coats, it was speculated that high concentrations of antigens like PGL-I in the plasma could influence the results of the antibody detection assay by in vivo complexing of antigens with some or all of the serum antibodies. Previous investigators have demonstrated that the 3,6-di-o-methyl-B-D-glucopyranose is the hapten determinant of the species-specific glycolipid. ${ }^{15-17}$ Therefore, due to the possible monovalent nature of PGL-I upon antigen antibody interaction one would not expect the formation of a lattice as commonly seen in precipitating immune complexes. This may explain our difficulty in demonstrating immune complexes using polyethylene glycol (data not shown). Consequently, we analysed the plasma samples for the presence of PGL-I. Analysis of selected plasma samples of 8 representative animals, 3 from Group A and 5 from Group B, indicated the presence of PGL-I in the plasma of 6 out of 8 animals. This was based on the migration pattern of the extracted component on TLC as compared with that of standard PGL-I and confirmed by its immunoreactivity with MAB F8b4 using ELISA. The presence of PGL-I in plasma was observed primarily in samples collected during the terminal phase of disease and correspondingly at a time in which a drop in IgG anti-PGL-I absorbance value was observed. Therefore, it is believed that this drop in absorbance value may be due to formation of complexes between high affinity IgG molecules and PGL-I in the plasma. This may be viewed as invivo antigen excess in which the degree of drop in absorbance value may vary based on the amount of antigen bound to antibody prior to the application of plasma to the antibody detection assay. A significant drop in absorbance values for IgM anti-PGL-I antibodies was not readily observed among animals in Group A and B. A possible explanation could be that there is a higher concentration of high affinity IgG anti-PGL-I molecules than the low affinity IgM antiPGL-I molecules.

Finally, the ability to detect $M$. leprae antigens like PGL-I in biological samples such as plasma may provide a potentially useful tool for the diagnosis of lepromatous leprosy, but such antigens do not appear promissory for early detection of disease in armadillos because they are usually abundant at a time in which clinical signs and symptoms are about to take place. Presence of PGL-I in plasma of human patients was also reported ${ }^{18,19}$ and its significance in detection of subclinical leprosy has yet to be determined.

The plasma of selected animals in Group A and B were further analysed for antibodies of $\mathrm{IgM}$ and $\mathrm{IgG}$ class to sonicated $M$. leprae components by means of immunoblotting. The predominant IgM and IgG responses were directed toward the M.leprae components with molecular weights ranging from $21-$ to $45-\mathrm{kDa}$. Similar observations, particularly with respect to the material with migration pattern at the region of $33-\mathrm{kDa}$, was also reported in lepromatous patients. ${ }^{4}$ The sera from armadillos having systemic infection with $M$. leprae were also shown to react significantly with $33-\mathrm{kDa}$ component. ${ }^{4,5} \mathrm{We}$ have previously shown that the components with broad diffuse staining bands are glycoprotein in nature. ${ }^{5}$ Others have also shown, upon electrophoresis and immunoblotting, that soluble fractions of disrupted $M$. leprae produced a major antigen with an apparent molecular mass of 30 - to $50-\mathrm{kDa} .^{7,20,21}$ This product has now been identified as LAM. ${ }^{22}$ 
Based on our observations and those of others, it appears that the major armadillo antibody activities are directed against LAM. This could reflect the relative immunodominance and/or accessibility of LAM in intact bacilli. Furthermore, the detection of antibodies to LAM in the plasma of armadillos prior to their inoculation with M. leprae is suggestive of the ubiquitous nature of the antigen.

It is usually difficult to verify whether the $33-\mathrm{kDa}$ glycoprotein is a single substance or a mixture of 2 or 3 substances with similar molecular weights. Based on the IgG immunoblot results seen in Figure 4, it appears that beneath this common broad diffuse band of immunoreactivity at the region of 33-kDa, there are as many as 3 distinct bands of immunoreactivity. As usually seen in IgM-type response (Figure 4), these components were not readily observed. A typical example of one such molecule is the $28-\mathrm{kDa}$ protein. Recently, the gene for a $28-\mathrm{kDa}$ protein of $M$. leprae has been cloned and was shown to be an important target of the humoral response in lepromatous leprosy. ${ }^{23}$ Further analysis of these components may prove important in the immunology of leprosy.

The $65-\mathrm{kDa}$ protein was also recognized as antibody evoking immunogen in armadillos. Although in our previous study, ${ }^{5}$ we have shown the ability of this molecule in inducing antibody of IgG class, most animals analysed here had antibody of IgM class to this molecule. However, the one animal with antibody activity to multiple components of $M$. leprae also had a very intense band of $\mathrm{IgG}$ immunoreactivity to the $65-\mathrm{kDa}$ protein. These observations suggest the individual animal variation in antibody response to the 65-kDa molecule. Also, antibody responses of armadillos to the IIIE9 epitope of the 65kDa protein of $M$. leprae have been analysed using a competition binding assay. This assay incorporated crude cell wall extract of $M$. leprae or purified recombinant $65-\mathrm{kDa}$ as antigen source. This epitope did not appear to be immunogenic. ${ }^{24}$ Finally, attempts were made to define lymphocyte blast transformation differences in high and low IgG PGL-I responders. However, the results were highly variable within and between groups (data not shown). In conclusion, as shown here, armadillo antibody activity increased significantly with time postinoculation and this was directed primarily toward LAM as well as PGL-I. Furthermore, all animals, regardless of their ability in mounting an elevated level of IgG anti-PGL-I response, showed a similar longitudinal pattern of IgG and $\operatorname{IgM}$ response to sonicated $M$. leprae components.

\section{Acknowledgments}

This work was supported in part by grants from the American Leprosy Missions and the Hansen's Disease Foundation. The authors wish to express their appreciation to personnel of the Microbiology Research Department for making available the armadillo plasma samples. We are also grateful to Mr Melvyn Morales for the computer analysis, Mr Sterling Christy for processing and storing the plasma samples, and to Ms Rosemary Hauge for secretarial assistance.

\section{References}

1 Ridley DS, Jopling WH. A classification of leprosy for research purposes. Lepr Rev, 1962; 33: 119-28.

${ }^{2}$ Kirchheimer WF, Storrs, EE. Attempts to establish the armadillo (Dasypus novemcinctus) as a model for the study of leprosy. Int J Lepr, 1971; 39: 693-702. 
3 Job CK, Sanchez RM, Hastings RC. Manifestations of experimental leprosy in the armadillos. Am J Trop Med Hyg 1985; 34: 151-61.

${ }^{4}$ Chakrabarty AK, Maire MA, Lambert PH. SDS-PAGE analysis of $M$. leprae protein antigens reacting with antibodies from sera from lepromatous patients and infected armadillos. Clin exp Imm, 1982; 49: 523-31.

5 Vadiee AR, Shannon EJ, Gillis TP, Hastings RC. Partial characterization of antigens from M. leprae evoking IgG and IgM antibodies in armadillos. Int J Lepr, 1988; 56(2): 274-82.

6 Harboe M, Closs O, Rees RJW, Walsh GP. Formation of antibody against Mycobacterium leprae antigen 7 in armadillos. J Med Microbiol, 1978; 11: 525-35.

7 Praputpitlaya K, Ivanyi J. Detection of an antigen (Ny4) common to M. tuberculosis and M. leprae by 'Tandem' immunoassay. J Immunol Methods, 1985; 79: 149-57.

8 Cho SN, Yanagihara DL, Hunter SW, Gelber RH, Brennan PJ. Serological specificity of phenolic glycolipidI from Mycobacterium leprae and use in serodiagnosis of leprosy. Infect Immun, 1983; 41: 1077-83.

9 Truman RW, Shannon EJ, Hagsted HV, Hugh-Jones ME, Wolff A, Hastings RC. Evaluation of the origin of Mycobacterium leprae infections in the wild armadillos, Dasypus novemcinctus. Am J Trop Hyg, 1986; 35: 588-93.

10 Vadiee AR, Shannon EJ, Gillis TP, Mshana RN, Hastings RC. Armadillo IgG and IgM antibody responses to phenolic glycolipid-1 during experimental infection with M. leprae. Int J Lepr, 1988; 56(3): 422-7.

11 Laemmli UK. Cleavage of structural proteins during the assembly of the head of the bacteriophage $\mathrm{T}_{4}$. Nature, 1970; 227: 680-9.

12 Hunter Shirley W, Fujiwara Tsuyoski, Brennan PJ. Structure and antigenicity of the major specific glycolipid antigen of Mycobacterium leprae. J Biol Chem, 1982; 257: 15072-8.

13 Levis WR, Meeker HC, Schuller-Levis G, Sessen E, Schwarer B. IgM and IgG antibodies to phenolic glycolipid-I from Mycobacterium leprae in leprosy: Insight into patient monitoring, erythema nodosum leprosum, and bacillary persistence. J Invest Dermatol, 1986; 86(5):529-34.

14 Gormus BJ, Ohaski DK, Ohkawa S, Walsh GP, Meyers WM, Brennan PJ, Trygg C. Serological responses to Mycobacterium leprae-specific phenolic glycolipid-I antigen in sooty Mangabey monkeys with experimental leprosy. Int J Lepr 1988; 56(4): 537-45.

15 Cho SN, Fujiwara T, Hunter SW, Rea TH, Gelber RH, Brennan PJ. Use of an artificial antigen containing the 3,6-di-o-methyl-B-D-glycopyranosyl epitope for the serodiagnosis of leprosy. J Infect Dis, 1984; 150(3): $311-22$.

16 Fujiwara T, Hunter SW, Cho SN, Aprinall GO, Brennan PJ. Chemical synthesis and serology of the disaccharides and trisaccharides of phenolic glycolipid antigens from the leprosy bacillus and preparation of a disaccharide protein conjugate for serodiagnosis of leprosy. Infect Immun, 1984; 43: 245-52.

17 Hunter SW, Brennan PJ. Further specific extracellular phenolic glycolipid antigens and a related diacylphthiocerol from Mycobacterium leprae. J Biol Chem, 1983; 258: 7556-62.

18 Cho SN, Hunter SW, Gelber RH, Rea TH, Brennan PJ. Quantitation of the phenolic glycolipid of Mycobacterium leprae and relevance to glycolipid antigenemia in leprosy. J Infect Dis, 1986; 153(3): 560-9.

19 Young DB, Harnisch JP, Knight J, Buchanan TM. Detection of phenolic glycolipid-I in sera from patients with lepromatous leprosy. J Infect Dis, 1985; 152(5): 1078-81.

20 Britton WJ, Hellquist L, Besten A, Raison RL. Mycobacterium leprae antigens involved in human immune response. J Immunol, 1985; 135: 4171-7.

${ }^{21}$ Ivanyi J, Sinha S, Aston R, Cussell D, Keen M, et al. Definition of species-specific and cross-reactive antigenic determinants of Mycobacterial leprae using monoclonal antibodies. Clin exp Imm, 1983; 52: 528-36.

${ }^{22}$ Hunter SW, Gaylord H, Brennan PJ. Structure and antigenicity of the phosphorylated lipopolysaccharide antigens from the leprosy and tubercule bacilli. J Biol Chem, 1986; 261: 12345-51.

${ }^{23}$ Cherayil BJ, Young RA. A 28-kDa protein from Mycobacterium leprae is a target of the human antibody response in lepromatous leprosy. J Immunol 1988; 141(12): 4370-5.

${ }^{24}$ Vadiee AR, Gillis TP, Shannon EJ. Confirmation of a false positive result associated with a competition inhibition assay used for detecting antibodies to a protein epitope of Mycobacterium leprae. Clin exp Imm, 1990; 79: 397-402. 